Trauma Surgery \& Acute Care Open

\title{
Predictive factors of mortality after colectomy in ischemic colitis: an ACS-NSQIP database study
}

\author{
Joshua Tseng, Brandi Loper, Monica Jain, Azaria V Lewis, Daniel R Margulies, \\ Rodrigo F Alban
}

Department of Surgery, Division of Acute Care Surgery, Trauma and Critical Care, Cedars-Sinai Medical Center, Los Angeles, California, USA

\section{Correspondence to}

Dr Rodrigo F Alban, Department of Surgery, Division of Acute Care Surgery, Trauma and Critical Care, Cedars-Sinai Medical Center, Suite 8215N, Los Angeles, CA 90048, USA; rodrigo.alban@cshs.org

This manuscript was previously presented at the 88th Annual Meeting of the Pacific Coast Surgical Association on February 18, 2017.

Received 10 August 2017 Revised 26 September 2017 Accepted 3 October 2017
To cite: Tseng J, Loper B, Jain M, et al. Trauma Surg Acute Care Open 2017;2:16.

\section{ABSTRACT}

Background Surgical intervention for ischemic colitis is associated with significant postoperative morbidity and mortality. Predictive factors of adverse outcomes have been reported in the literature, but are based on small sample populations. We sought to identify risk factors for mortality after emergent colectomy for ischemic colitis using a clinical outcomes database.

Methods The American College of Surgeons National Surgical Quality Improvement Project database was queried from 2010 to 2015 to identify emergent colectomies performed for ischemic colitis using Current Procedural Terminology and International Classification of Diseases, Ninth Revision codes. Univariate and multivariate logistic regression analysis was used to identify independent risk factors associated with increased risk of mortality.

Results A total of 4548 patients undergoing emergent colectomies for ischemic colitis were identified. Overall, 30-day postoperative mortality was $25.3 \%$. On univariate analysis, preoperative risk factors associated with a higher rate of mortality include dyspnea, functional status, ventilator dependency, history of chronic obstructive pulmonary disease, ascites, congestive heart failure exacerbation, hypertension, dialysis dependency, cancer, open wounds, chronic steroids, weight loss $>10 \%$, transfusions within 72 hours before surgery, septic shock and duration from hospital admission to surgery. Factors that were significant for mortality on logistic regression analysis include elderly age, poor functional status, multiple comorbidities, septic shock, blood transfusion, acute renal failure and the duration of time from hospital admission to surgery. Conclusions Postoperative morbidity and mortality rates for ischemic colitis remain significantly high. Identification of risk factors may help patient selection for surgical interventions, and make informed decisions with patients and family members. Although it is certainly challenging, early diagnosis and prompt surgical intervention for patients with ischemic colitis may improve outcomes.

Study type and level of evidence Therapeutic/care management, level II

\section{INTRODUCTION}

Ischemic colitis is a common and potentially lethal disease of the gastrointestinal tract with high rates of postoperative morbidity and mortality. Its clinical presentation is variable and often non-specific, and its course ranges from mild self-limiting forms to gangrene, perforation and intra-abdominal sepsis, requiring surgical intervention. ${ }^{1}$ Without signs that prompt escalation of care, such as gastrointestinal bleeding, peritonitis or worsening acidosis, early diagnosis and intervention can be challenging. ${ }^{23}$

Approximately 14\%-66\% of patients diagnosed with ischemic colitis require surgery, ${ }^{2-6}$ with up to two-thirds of these patients undergoing an emergent operation. ${ }^{7}$ Postoperative mortality rates are significant, ranging from $5 \%$ to over $80 \% .^{2568-10}$ This wide variation in mortality has been studied by others, who have identified some risk factors including advanced age, male gender, coronary artery disease, atrial fibrillation, recent cardiovascular surgery and history of dialysis. ${ }^{1} 34679-11$ Clinical predictors of mortality include abdominal tenderness, signs of peritonitis, absence of gastrointestinal bleeding, intraperitoneal free fluid, lactic or metabolic acidosis, preoperative hemodynamic instability, perioperative vasopressors, need for mechanical ventilation and surgical delay $>3$ days. $^{3} 46810-13$ Other studies also identified laterality of ischemia, extent of surgical resection and etiology of ischemia on specimen pathology to be predictive of mortality as well. ${ }^{9}$

Studies on patients undergoing colectomies for ischemic colitis have been generally limited to small, retrospective, single-center studies with $<200$ cases per study. These studies also combined both emergent and non-emergent operations. Considering the significant adverse outcomes associated with ischemic colitis, we sought to identify predictive risk factors for mortality and complications after emergent colectomy for ischemic colitis using the American College of Surgeons National Surgical Quality Improvement Project (ACS-NSQIP) database.

\section{METHODS}

Data collection

ACS-NSQIP is a national database of clinical information collected from participating hospitals. It contains blinded, risk-adjusted data regarding complication rates and surgical outcomes, and places hospitals against a national benchmark. Variables include preoperative risk factors, intraoperative variables and 30-day postoperative mortality and morbidity outcomes. The data are collected by trained surgical clinical reviewers by chart review, coded into variables with strict, rigorous definitions and then de-identified for public use.

The ACS-NSQIP databases from 2010 to 2015 were filtered for patients undergoing emergent surgery. A total of 4548 colectomies performed for ischemic colitis were identified. Current Procedural Terminology (CPT) codes were used to identify 
colectomies (CPT codes 44204, 44205, 44206, 44207, 44208, 44210, 44211, 44212, 44188, 44140, 44141, 44143, 44144, 44145, 44146, 44147, 44150, 44151, 44155, 544156, 44157, 44158, 44160, 44120, 44121, 44125, 44130). International Classification of Diseases, Ninth Revision (ICD-9) codes were used to identify the diagnosis of ischemic colitis (ICD-9-CM $557.0,557.1,557.9)$. From this population, preoperative demographic data were obtained, including age, gender, race, body mass index and comorbidities. Preoperative lab values and clinical variables such as days from admission to operation, renal failure, need for dialysis, emergency status and wound classification were obtained. Information regarding the type, laterality and extent of surgery were derived from CPT codes when unequivocal. Postoperative mortality and morbidities, such as cardiovascular, pulmonary, renal, septic and venous thromboembolic complications were analyzed. A comorbidity index was created by summing the unweighted number of comorbidities captured by the ACS-NSQIP database. Patients were divided into two cohorts-survivors and non-survivors-based on 30-day postoperative mortality.

\section{Statistical analyses}

Normally distributed continuous variables were described as mean \pm SD deviation, and non-normally distributed continuous variables were described by median and IQR. Categorical variables were listed in absolute numbers and percent prevalence (\%) in the study group. Statistical analyses were performed between survivors and non-survivors using the IBM SPSS software, V.22.0 (SPSS, Armonk, New York, USA). Independent two sample t-tests were used to assess normally distributed continuous variables for significance, and the Mann-Whitney $U$ test was used to assess non-normally distributed continuous and ordinal variables. Categorical variables were compared using the $\mathrm{X}^{2}$ test. After identifying variables that were statistically significant on univariate analysis, preoperative variables that were clinically relevant were analyzed with a multivariate logistic regression model.

\section{RESULTS}

\section{Patient characteristics}

A total of 4548 patients underwent an emergent colectomy with a primary diagnosis of ischemic colitis in the 2010-2015 ACS-NSQIP database. Demographic characteristics are listed in table 1 . Of all patients, $42.5 \%$ were male and $57.4 \%$ were female. The majority of patients were elderly, with $22.5 \%$ older than $80 \%$ and $41.6 \%$ aged $65-80$ (data not shown); $78.4 \%$ were white, $11.0 \%$ were black, $1.4 \%$ were Asian, 0.6\% were American Indian/Alaskan, $0.2 \%$ were Hawaiian/Pacific Islander and $8.4 \%$ were listed as 'other'. There were no significant differences between survivors and non-survivors in terms of race and ethnicity.

Operatively, the majority of cases were performed open $(95.7 \%)$ as opposed to laparoscopic $(4.3 \%)$ per CPT code (table 1). Most cases involved partial colectomies (84.4\%) compared with total (15.6\%). Sixty-four per cent of partial colectomies involved the right colon, and 36\% involved the left. A primary anastomosis was performed in $63 \%$ of cases, while an ostomy was created in $35.5 \%$ of cases; $1.5 \%$ of cases had both an anastomosis and an ostomy done in the initial operation. The rates of valid data are listed in table 1.

Compared with patients in the survivor cohort, non-survivors were older $(72.0 \pm 12.6$ vs $67.3 \pm 14.7, \mathrm{p}<0.001)$, and had higher rates of dyspnea at rest and moderate exertion $(12.6 \%$ vs $5.1 \%$ and $11.8 \%$ vs $9.0 \%, \mathrm{p}<0.001$, respectively), total or partial functional dependency $(14.7 \%$ vs $5.5 \%$ and $14.5 \%$ vs $9.8 \%, \mathrm{p}<0.001$, respectively), ventilator dependency $(38.5 \%$ vs $11.2 \%, \mathrm{p}<0.001)$, history of severe chronic obstructive pulmonary disease $(25.1$ vs $15.7 \%, \mathrm{p}<0.001)$, ascites $(8.3 \%$ vs $5.1 \%, \mathrm{p}<0.001)$, congestive heart failure exacerbation within 30 days of surgery $(11.0 \%$ vs $5.3 \%, \mathrm{p}<0.001)$, hypertension requiring medications $(77.0 \%$ vs $70.2 \%, \mathrm{p}<0.001)$, acute renal failure within 24 hours prior to surgery $(14.0 \%$ vs $7.8 \%$, $\mathrm{p}<0.001)$, dialysis 2 weeks prior to surgery $(18.1 \%$ vs $8.9 \%$, $\mathrm{p}<0.001)$, disseminated cancer $(4.0 \%$ vs $2.3 \%, \mathrm{p}<0.001)$, open or infected wounds $(9.7 \%$ vs $5.6 \%, \mathrm{p}<0.001)$, chronic steroid use $(11.1 \%$ vs $8.7 \%, \mathrm{p}=0.013)$, weight loss $>10 \%(6.7 \%$ vs $3.5 \%, \mathrm{p}=0.013)$, transfusions within 72 hours before surgery $(15.9 \%$ vs $8.0 \%, \mathrm{p}<0.001)$ and preoperative septic shock (52.2\% vs $23.1 \%, \mathrm{p}<0.001)$.

Non-survivors were more likely to have an open operation $(98.5 \%$ vs $94.6 \%, \mathrm{p}<0.001)$ and a total colectomy $(20.3 \%$ vs $13.8 \%, \mathrm{p}<0.001)$. There was no significant difference in terms of laterality $(p=0.392)$. The rate of ostomy creation versus primary anastomosis did not differ between cohorts $(p=0.944)$.

\section{Postoperative outcomes}

The 30-day postoperative mortality rate following emergent colectomy for ischemic colitis was $25.3 \%$. Comparisons of postoperative complications categorized by survivors and non-survivors are shown in table 2 . The most common complications were prolonged intubation (35.2\%), septic shock (26.3\%) and pneumonia (13.5\%). Compared with survivors, non-survivors had a higher rate of respiratory complications, such as pneumonia $(16.5 \%$ vs $12.5 \%, \mathrm{p}<0.001)$, unplanned intubation $(15.6 \%$ vs $9.2 \%, \mathrm{p}<0.001)$ and prolonged intubation $>48$ hours (46.1\% vs $31.5 \%, \mathrm{p}<0.001)$. Non-survivors had higher rates of septic shock $(46.6 \%$ vs $19.4 \%, \mathrm{p}<0.001)$ and lower rates of sepsis $(7.0 \%$ vs $11.3 \%, \mathrm{p}<0.001)$. Renal complications were also higher, including acute renal failure $(13.0 \%$ vs $3.7 \%, \mathrm{p}<0.001)$. Rates of cerebrovascular accident, cardiac arrest and myocardial infarction were higher in non-survivors $(3.3 \%$ vs $1.5 \%$, $\mathrm{p}<0.001,15.6 \%$ vs $1.2 \%, \mathrm{p}<0.001,4.9 \%$ vs $3.1 \%, \mathrm{p}<0.001$, respectively).

\section{Multivariate regression}

Table 3 reports the results of the multivariate logistic regression analysis. Factors associated with higher odds of 30-day mortality include age, comorbidity index, functional status, preoperative septic shock, preoperative renal failure, transfusions prior to surgery and days from hospital admission to surgery.

In our analysis, the OR for mortality progressively increased with advancing age. Patients aged 65-79 years had a $235 \%$ higher odds of mortality than those aged $<40$ years (OR 3.36, $95 \%$ CI 1.96 to 5.74 ), and patients aged 80 years or older had $413 \%$ higher odds of mortality (OR 5.13; 95\% CI 2.97 to 8.86). Like age, the odds of death increased with the number of comorbidities. Patients with four or more comorbidities had 345\% higher odds of mortality compared with those with none (OR 4.45, 95\% CI 3.06 to 6.47). Preoperative clinical factors such as septic shock, blood transfusions and acute renal failure also had increased odds of mortality (OR 2.8, 95\% CI 2.39 to 3.29; OR $1.4,95 \%$ CI 1.11 to 1.76 ; OR $3.06,95 \%$ CI 2.32 to 4.03 , respectively). Compared with patients who went immediately into surgery on the day of admission, those who went to surgery 1 or 2 days after admission had no increased odds of mortality (OR $0.99,95 \%$ CI 0.82 to 1.20 ; OR 1.04 , 95\% CI 0.78 to 1.38 ). 
Table 1 Preoperative and intraoperative characteristics of patients with ischemic colitis

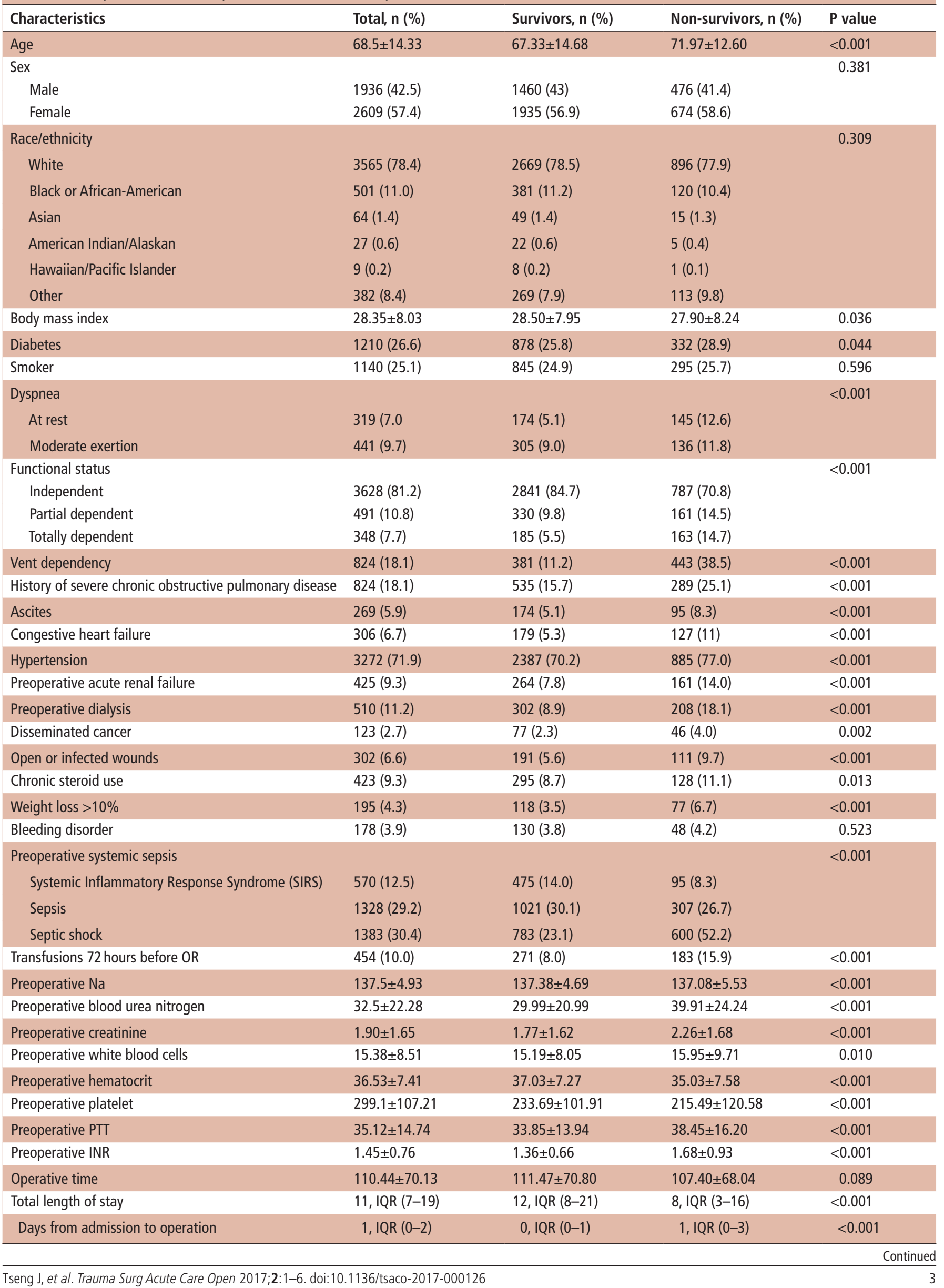




\begin{tabular}{|c|c|c|c|c|}
\hline Characteristics & Total, n (\%) & Survivors, n (\%) & Non-survivors, $\mathrm{n}(\%)$ & $P$ value \\
\hline \multicolumn{5}{|l|}{ Wound classification } \\
\hline One clean & $46(1.0)$ & $33(1.0)$ & $13(1.1)$ & $<0.001$ \\
\hline Two clean/contaminated & $1039(22.8)$ & $842(24.8)$ & $197(17.1)$ & \\
\hline Three contaminated & $1590(35.0)$ & $1252(36.8)$ & $338(29.4)$ & \\
\hline Four dirty/infected & $1873(41.2)$ & $1271(37.4)$ & $602(52.3)$ & \\
\hline American Society of Anesthesiologists classification & & & & $<0.001$ \\
\hline 1 & $33(0.7)$ & $33(1.0)$ & 0 & \\
\hline 2 & $322(7.1)$ & $312(9.2)$ & $10(0.9)$ & \\
\hline 3 & $1434(31.5)$ & $1284(37.8)$ & $150(13.0)$ & \\
\hline 4 & $2272(50.0)$ & $1556(45.8)$ & $716(62.3)$ & \\
\hline 5 & $476(10.5)$ & $210(6.2)$ & $266(23.1)$ & \\
\hline Surgical approach* & & & & $<0.001$ \\
\hline Ex-lap & $2763(95.7)$ & $1971(94.6)$ & $792(98.5)$ & \\
\hline Laparoscopic & $125(4.3)$ & $113(5.4)$ & $12(1.5)$ & \\
\hline Extent of colectomy $\dagger$ & & & & $<0.001$ \\
\hline Partial & $2437(84.4)$ & $1796(86.2)$ & $641(79.7)$ & \\
\hline Total & $451(15.6)$ & $288(13.8)$ & $163(20.3)$ & \\
\hline Laterality of colectomył & & & & 0.392 \\
\hline Right hemicolectomy & $753(64.0)$ & $558(63.3)$ & $195(66.1)$ & \\
\hline Left hemicolectomy & $423(36.0)$ & $323(36.7)$ & $100(33.9)$ & \\
\hline Ostomy or anastomosis§ & & & & 0.944 \\
\hline Ostomy only & $894(35.5)$ & $654(35.5)$ & $240(35.7)$ & \\
\hline Anastomosis only & $1585(63.0)$ & $1161(63.0)$ & $424(63.0)$ & \\
\hline Anastomosis with ostomy & $36(1.5)$ & $28(1.5)$ & $9(1.3)$ & \\
\hline
\end{tabular}

$* \mathrm{n}=2888,63.5 \%$.

$\mathrm{tn}=2888,63.5 \%$.

$\ddagger \mathrm{n}=1176,25.9 \%$.

$\S n=2516,55.3 \%$.

However, patients who went to surgery 3 days or later had a $39 \%$ higher odds of mortality (OR 1.39 , 95\% CI 1.14 to 1.70 ).

The model fit the observed data well as tested using the $\mathrm{C}$ statistic $(C=0.76)$ and the Hosmer-Lemeshow test $(p=0.549)$. The $\mathrm{C}$ statistics represents the area under the receiver-operating curve. In the Hosmer-Lemeshow test, $\mathrm{p}$ value $>0.05$ signifies that the model fits the observed data.

\section{DISCUSSION}

Ischemic colitis can present with a wide variety of clinical pictures, ranging from self-limiting observation to surgical intervention. Rates of surgical treatment vary throughout the literature, from $14 \%$ to $66 \%$ in published studies. ${ }^{2-6}$ Unfortunately, postoperative morbidity and mortality rates remain high, from $5 \%$ to over $80 \% .^{2568-10}$ On our analysis of ACS-NSQIP hospitals, patients undergoing emergent colectomy for ischemic colitis had a 30 -day postoperative mortality rate of $25.3 \%$. This number lies within range of previously reported rates in smaller sample populations. Patients also had high rates of postoperative complications including prolonged intubation (35.2\%), pneumonia (13.5\%), septic shock $(26.3 \%)$ and acute renal failure $(6.0 \%)$. These findings highlight the severity of the disease and its dismal outcomes after surgical intervention.

Prior studies have identified multiple risk factors predictive of mortality, such as advanced age, male sex, history of coronary artery disease and atrial fibrillation, history of peripheral vascular disease, prior cardiovascular surgery and dialysis dependence. ${ }^{134679-11}$ However, these studies were generally retrospective, single center, based on sample sizes of $<200$, and included both emergent and elective operations. We performed an analysis of the ACS-NSQIP database based on a large, robust sample population from over 600 hospitals across the nation, and selectively analyzed patients who underwent emergent surgery. In our study, patient characteristics significant for mortality on multivariate analysis include elderly age, poor functional status, multiple comorbidities, preoperative acute renal failure, preoperative septic shock, preoperative blood transfusion and notably, duration of time from hospital admission to surgery.

In the ACS-NSQIP population, operating on patients 3 days or later after their presentation to the hospital resulted in a $39 \%$ higher odds of 30-day postoperative mortality. This finding has rarely been studied in published literature; in a retrospective study of 50 patients, Noh et al demonstrated a $73.3 \%$ mortality rate vs $34.3 \%$ mortality rate when surgery was delayed for $>3$ days. ${ }^{10}$ Unfortunately, due to the inherent limitations of the database, we cannot tell if this is a delay in diagnosis, surgical consultation or surgical intervention. Regardless, this finding highlights the importance of early diagnosis for ischemic colitis. Prompt surgical intervention is warranted, and may even prevent clinical deterioration into septic shock and renal failure, which are factors that also conferred increased odds of mortality in our study. The duration of time from hospital admission to surgery 
Table 2 30-day outcomes of colectomy for ischemic colitis

\begin{tabular}{|c|c|c|c|c|}
\hline & $\begin{array}{l}\text { Total, } \\
\text { n (\%) } \\
\end{array}$ & $\begin{array}{l}\text { Survivors, } \\
\mathrm{n}(\%)\end{array}$ & $\begin{array}{l}\text { Non-survivors, } \\
\mathrm{n}(\%)\end{array}$ & $P$ value \\
\hline Mortality & $1150(25.3)$ & - & - & - \\
\hline In-hospital & $945(20.8)$ & - & - & - \\
\hline \multicolumn{5}{|l|}{ Wound complications } \\
\hline Superficial SSI & $242(5.3)$ & $229(6.7)$ & $13(1.1)$ & $<0.001$ \\
\hline Deep SSI & $79(1.7)$ & $68(2.0)$ & $11(1.0)$ & 0.019 \\
\hline Organ/space SSI & $269(5.9)$ & $210(6.2)$ & $59(5.1)$ & 0.192 \\
\hline Wound disruption & $117(2.6)$ & $101(3.0)$ & $16(1.4)$ & 0.003 \\
\hline \multicolumn{5}{|l|}{ Respiratory complications } \\
\hline Pneumonia & $614(13.5)$ & $424(12.5)$ & $190(16.5)$ & 0.001 \\
\hline Unplanned intubation & $502(11.0)$ & $312(9.2)$ & $190(16.5)$ & $<0.001$ \\
\hline Vent $>48$ hours & $1599(35.2)$ & $\begin{array}{r}1069 \\
(31.5)\end{array}$ & $530(46.1)$ & $<0.001$ \\
\hline \multicolumn{5}{|l|}{ Renal complications } \\
\hline $\begin{array}{l}\text { Progressive renal } \\
\text { insufficiency }\end{array}$ & $101(2.2)$ & $73(2.1)$ & $28(2.4)$ & 0.569 \\
\hline Acute renal failure & $275(6.0)$ & $125(3.7)$ & $150(13.0)$ & $<0.001$ \\
\hline UTI & $206(4.5$ & $171(5.0)$ & $35(3.0)$ & 0.005 \\
\hline \multicolumn{5}{|c|}{ Cardiovascular complications } \\
\hline Pulmonary embolism & $39(0.9)$ & $37(1.1)$ & $2(0.2)$ & 0.004 \\
\hline Stroke/CVA & $88(1.9)$ & $50(1.5)$ & $38(3.3)$ & $<0.001$ \\
\hline Cardiac arrest & $221(4.9)$ & $42(1.2)$ & $179(15.6)$ & $<0.001$ \\
\hline Myocardial infarction & $163(3.6)$ & $107(3.1)$ & $56(4.9)$ & 0.007 \\
\hline DVT & $154(3.4)$ & $125(3.7)$ & $29(2.5)$ & 0.061 \\
\hline $\begin{array}{l}\text { Postoperative } \\
\text { bleeding/transfusions }\end{array}$ & $1725(37.9)$ & $1103(32.5)$ & $622(54.1)$ & $<0.001$ \\
\hline \multicolumn{5}{|l|}{ Sepsis complications } \\
\hline Sepsis & $465(10.2)$ & $384(11.3)$ & $81(7.0)$ & $<0.001$ \\
\hline Septic shock & $1194(26.3)$ & 658 (19.4) & $536(46.6)$ & $<0.001$ \\
\hline Return to $\mathrm{OR}$ & $652(14.3)$ & $463(13.6)$ & $189(16.4)$ & 0.019 \\
\hline $\begin{array}{l}\text { Unplanned } \\
\text { reoperation }\end{array}$ & $364(11.7)$ & $264(11.3)$ & $100(12.8)$ & 0.266 \\
\hline
\end{tabular}

CVA, cerebrovascular accident; DVT, deep vein thrombosis; SSI, surgical site infection; UTI, urinary tract infection.

represents a potentially modifiable risk factor that should be strongly emphasized and further studied.

Laterality and extent of ischemia have previously been identified as predictors of postoperative mortality. For example, a study by Genstorfer et al found a 3.8 and 11 times OR for death in right-sided colitis and pan colitis compared with left, respectively. ${ }^{9}$ Longstreth and Yao found a 14.6 times OR for death in right-sided and bilateral colitis together when compared with left-sided colitis. ${ }^{3}$ In contrast, our large sample database study showed that there was no significant difference in laterality of colectomy between survivors and non-survivors. However, non-survivors were also more likely to have total colectomies compared with survivors. Overall, our study findings are more robust with their larger sample size, but are limited by the extrapolation of laterality and extent of surgical resection from CPT code as opposed to the operative report.

Similar to prior studies, elderly patients with poor functional status and multiple comorbidities had higher rates of death. ${ }^{35614-16}$ Those older than 80 years carried the worst prognosis of all factors assessed in the database, with $413 \%$ higher odds of mortality. The odds of mortality increased progressively with the number of comorbidities present, up to $345 \%$ higher
Table 3 Multivariate model showing predictive factors for 30-day mortality following colectomy for ischemic colitis

\begin{tabular}{|c|c|c|c|c|}
\hline \multirow[b]{2}{*}{ Variable } & \multirow[b]{2}{*}{ OR } & \multicolumn{2}{|l|}{$95 \% \mathrm{Cl}$} & \multirow[b]{2}{*}{$P$ value } \\
\hline & & Lower & Upper & \\
\hline \multicolumn{5}{|l|}{ Age group (years) } \\
\hline $18-40$ & Reference & & & \\
\hline $41-64$ & 1.68 & 0.98 & 2.90 & 0.061 \\
\hline $65-79$ & 3.36 & 1.96 & 5.74 & $<0.001$ \\
\hline$>80$ & 5.13 & 2.97 & 8.86 & $<0.001$ \\
\hline \multicolumn{5}{|l|}{ Comorbidities } \\
\hline 0 & Reference & & & \\
\hline 1 & 1.76 & 1.23 & 2.51 & 0.002 \\
\hline 2 & 2.65 & 1.87 & 3.76 & $<0.001$ \\
\hline 3 & 3.10 & 2.15 & 4.47 & $<0.001$ \\
\hline$>4$ & 4.45 & 3.06 & 6.47 & $<0.001$ \\
\hline \multicolumn{5}{|l|}{ Functional status } \\
\hline Independent & Reference & & & \\
\hline Partially dependent & 1.29 & 1.03 & 1.61 & 0.026 \\
\hline Fully Dependent & 1.65 & 1.28 & 2.13 & $<0.001$ \\
\hline Septic shock (preoperative) & 2.80 & 2.39 & 3.29 & $<0.001$ \\
\hline $\begin{array}{l}\text { Transfusions } \\
\text { (preoperative) }\end{array}$ & 1.40 & 1.11 & 1.76 & 0.005 \\
\hline Renal failure (preoperative) & 3.06 & 2.32 & 4.03 & $<0.001$ \\
\hline \multicolumn{5}{|l|}{ Days to surgery } \\
\hline 0 & Reference & & & \\
\hline 1 & 0.99 & 0.82 & 1.20 & 0.939 \\
\hline 2 & 1.04 & 0.78 & 1.38 & 0.795 \\
\hline$>3$ & 1.39 & 1.14 & 1.70 & 0.001 \\
\hline
\end{tabular}

in patients with four or more comorbidities. Interestingly, our study showed that male gender was not a significant predictor of mortality $(p=0.381)$. This stands in contrast to other small, single-center studies by Hughier et al and Longstreth and Yao that found higher rates of death with males. ${ }^{36}$ Although these factors are not modifiable, this knowledge can be used to aid key stakeholders to make informed decisions regarding optimal surgical care.

The lack of gastrointestinal bleeding has been identified as a risk factor for death in multiple previous publications. ${ }^{5616}$ For example, studies by Hughier et al, Paterno et al, Moszkowicz et al and Añón et al found that the absence of rectal bleeding was a risk factor for surgery, delayed operation and death. ${ }^{561117}$ Likewise, the presence of rectal bleeding was protective and conferred better outcomes per Longstreth and Yao. ${ }^{3}$ On the other hand, our study demonstrated that patients who received blood transfusions within 72 hours before surgery had higher rates of postoperative death; this has also been described in other studies. ${ }^{7}$ These findings appear to be incongruent. Unfortunately, the ACS-NSQIP database does not capture information regarding preoperative gastrointestinal bleeding, nor does it describe indications for blood transfusion, for example, massive gastrointestinal bleeding, shock or low hemoglobin and hematocrit levels after a lengthy hospital course. One explanation is that patients without evidence of frank gastrointestinal bleeding have delays in diagnosis and surgical intervention; blood transfusions may also represent a delay in diagnosis, prolonged hospital course or hemorrhagic shock, all of which led to poor outcomes.

The strength of our study stems from the ACS-NSQIP database's robust sample size and high-quality data from multiple 
hospitals nationwide. However, there are significant limitations to this study. First, our study is retrospective. Inaccurate coding with ICD-9 code for diagnosis or CPT code for surgery can result in cases erroneously added in or omitted from the database. Furthermore, in our study, results regarding laterality and extent of surgery are extrapolated from CPT code and highly susceptible to this type of error. Definitions of certain variables, such as preoperative acute renal failure and postoperative acute kidney injury, differ from other consensus definitions and have been shown to underestimate the actual incidence and risk associated with each condition. ${ }^{18}$ In addition, many clinical variables used in the assessment of ischemic colitis are not captured. These include variables such as physical exam findings, recent cardiac or vascular surgical history, ${ }^{9}{ }^{19-22}$ atrial fibrillation, ${ }^{4} 911$ radiographic findings, colonoscopy results, evidence of gastrointestinal bleeding, hemorrhagic or hypovolemic shock, lactate levels ${ }^{4911} 13$ and the duration and severity of vasopressor use. ${ }^{689}$ These clinically relevant factors should be studied further with large sample populations.

\section{CONCLUSIONS}

Independent risk factors of mortality after emergent colectomy for ischemic colitis include elderly age, poor functional status, multiple comorbidities, preoperative septic shock, preoperative blood transfusions, preoperative acute renal failure and delay from hospital admission to surgery. Identification of these risk factors may aid surgeons in patient selection for surgery, and making informed decisions with key stakeholders. Although it can be challenging to make an early diagnosis in ischemic colitis, prompt surgical intervention may improve outcomes.

Contributors Conception or design of the work: JT, BL, MJ, RFA. Data collection: JT, BL, MJ, AVL, RFA. Data analysis and interpretation: JT, BL, MJ, AVL, RFA. Drafting the article: JT, BL, AVL, RFA. Critical revision of the article: JT, BL, DRM, RFA. Final approval of the version to be published: DRM, RFA.

Competing interests None declared.

Ethics approval According to our institution's Office of Research Compliance and Quality Improvement, while the activity described in our study proposal involves research, it does not meet the definition of 'human subject research' as defined in the DHHS (45 CFR 46) or FDA regulations (21 CFR 50) for the protection of human subjects. The use of materials in our study proposal meets the definition of 'acceptably derived'.

Provenance and peer review Not commissioned; externally peer reviewed.

Data sharing statement The data from this study were obtained from the ACS-NSQIP Participant-use Files, and are available after agreeing to comply with the Data-use Agreement at the following website: https://www.facs.org/qualityprograms/acs-nsqip/program-specifics/participant-use

Open Access This is an Open Access article distributed in accordance with the Creative Commons Attribution Non Commercial (CC BY-NC 4.0) license, which permits others to distribute, remix, adapt, build upon this work non-commercially, and license their derivative works on different terms, provided the original work is properly cited and the use is non-commercial. See: http://creativecommons.org/ licenses/by-nc/4.0/ (c) Article author(s) (or their employer(s) unless otherwise stated in the text of the article) 2017. All rights reserved. No commercial use is permitted unless otherwise expressly granted.

\section{REFERENCES}

1 Moszkowicz D, Mariani A, Trésallet C, Menegaux F. Ischemic colitis: the ABCs of diagnosis and surgical management. J Visc Surg 2013;150:19-28.

2 Theodoropoulou A, Koutroubakis IE. Ischemic colitis: clinical practice in diagnosis and treatment. World J Gastroenterol 2008;14:7302-8.

3 Longstreth GF, Yao JF. Epidemiology, clinical features, high-risk factors, and outcome of acute large bowel ischemia. Clin Gastroenterol Hepatol 2009;7:1075-80.

4 Acosta-Merida MA, Marchena-Gomez J, Hemmersbach-Miller M, Roque-Castellano C, Hernandez-Romero JM. Identification of risk factors for perioperative mortality in acute mesenteric ischemia. World J Surg 2006;30:1579-85.

5 Moszkowicz D, Trésallet C, Mariani A, Lefevre JH, Godiris-Petit G, Noullet S, Rouby $\mathrm{JJ}$, Menegaux F. Ischaemic colitis: indications, extent, and results of standardized emergency surgery. Dig Liver Dis 2014;46:505-11.

6 Huguier M, Barrier A, Boelle PY, Houry S, Lacaine F. Ischemic colitis. Am J Surg 2006;192:679-84.

7 Antolovic D, Koch M, Hinz U, Schöttler D, Schmidt T, Heger U, Schmidt J, Büchler MW, Weitz J. Ischemic colitis: analysis of risk factors for postoperative mortality. Langenbecks Arch Surg 2008;393:507-12.

8 Castleberry AW, Turley RS, Hanna JM, Hopkins TJ, Barbas AS, Worni M, Mantyh CR, Migaly J. A 10-year longitudinal analysis of surgical management for acute ischemic colitis. J Gastrointest Surg 2013;17:784-92.

9 Genstorfer J, Schäfer J, Kettelhack C, Oertli D, Rosenthal R. Surgery for ischemic colitis: outcome and risk factors for in-hospital mortality. Int J Colorectal Dis 2014:29:493-503.

10 Noh M, Yang SS, Jung SW, Park JH, Im YC, Kim KY. Poor prognostic factors in patients who underwent surgery for acute non-occlusive ischemic colitis. World J Emerg Surg 2015;10:12.

11 Paterno F, McGillicuddy EA, Schuster KM, Longo WE. Ischemic colitis: risk factors for eventual surgery. Am J Surg 2010;200:646-50.

12 Chung JW, Cheon JH, Park JJ, Jung ES, Choi EH, Kim H. Development and validation of a novel prognostic scoring model for ischemic colitis. Dis Colon Rectum 2010;53:1287-94

13 Reissfelder C, Sweiti H, Antolovic D, Rahbari NN, Hofer S, Büchler MW, Weitz J, Koch M. Ischemic colitis: who will survive? Surgery 2011;149:585-92.

$14 \mathrm{Ma} \mathrm{X}, \mathrm{Ma} \mathrm{H}$, Zhu J, Liu J. Clinical characteristics of ischemic colitis in elderly patients. Hepatogastroenterology 2015;62:620-3.

15 Yadav S, Dave M, Edakkanambeth Varayil J, Harmsen WS, Tremaine WJ, Zinsmeister AR, Sweetser SR, Melton LJ, Sandborn WJ, Loftus EV. A population-based study of incidence, risk factors, clinical spectrum, and outcomes of ischemic colitis. Clin Gastroenterol Hepatol 2015:13:731-8.

16 Barouk J, Gournay J, Bernard P, Masliah C, Le Neel JC, Galmiche JP. [Ischemic colitic in the elderly: predictive factors of gangrenous outcome]. Gastroenterol Clin Biol 1999;23:470-4.

17 Añón R, Boscá MM, Sanchiz V, Tosca J, Almela P, Amorós C, Benages A. Factors predicting poor prognosis in ischemic colitis. World J Gastroenterol 2006;12:4875-8.

18 Bihorac A, Brennan M, Ozrazgat-Baslanti T, Bozorgmehri S, Efron PA, Moore FA, Segal MS, Hobson CE. National surgical quality improvement program underestimates the risk associated with mild and moderate postoperative acute kidney injury. Crit Care Med 2013:41:2570-83.

19 Schütz A, Eichinger W, Breuer M, Gansera B, Kemkes BM. Acute mesenteric ischemia after open heart surgery. Angiology 1998:49:267-73.

20 Sakorafas GH, Tsiotos GG. Intra-abdominal complications after cardiac surgery. Eur J Surg 1999:165:820-7.

21 Simić 0 , Strathausen S, Hess W, Ostermeyer J. Incidence and prognosis of abdominal complications after cardiopulmonary bypass. Cardiovasc Surg 1999;7:419-24.

22 Mangi AA, Christison-Lagay ER, Torchiana DF, Warshaw AL, Berger DL. Gastrointestinal complications in patients undergoing heart operation: an analysis of 8709 consecutive cardiac surgical patients. Ann Surg 2005;241:895-901. 